\title{
Waveform Similarity-Based Robust Pilot Protection for Transmission Lines
}

\author{
Guangxiao Zhang, Student Member, IEEE, Xiaoyang Tong, Qiteng Hong, Member, IEEE, and \\ Campbell D. Booth
}

\begin{abstract}
This paper proposes a robust pilot protection scheme based on waveform similarity that restricts the influence of outliers in fault line identification. By comparing two refactoring currents constructed using the time-domain sampling values of currents at the two terminals of a transmission line, a waveform similarity algorithm called the Kendall's Tau coefficient (KTC) is employed to distinguish two different datasets of a protected transmission line. A reliable fault identification is realized under different fault types, distances, and resistances. Proposed criteria that are robust against to current transformer saturation, synchronization error, and noise are proposed. The KTC is based on simply the rank order instead of the metric value and thus limits outliers to a moderate level, ensuring protection safety in cases where outliers that cannot be eliminated completely are included in the current waveforms. The simulation results demonstrate the excellent performance of the proposed robust pilot protection scheme.
\end{abstract}

Index Terms-Similarity comparison, Kendall's Tau coefficient, outliers, cyber-security, pilot protection.

\section{INTRODUCTION}

$\mathrm{W}$ ITH the increasing power demand, transmission lines are operated near their stability limits. Fast and reliable fault clearance in a transmission line is vital for reducing electrical equipment stress, enhancing system stability, and improving power quality [1]-[3]. Pilot protection plays a vital role in a transmission network because of its high speed, sensitivity, and selectivity in fault detection [4]. However, conventional pilot protection technologies face some challenges, particularly concerning current transformer (CT) saturation, high fault resistance, synchronization error, and noise. Therefore, the development of reliable protection schemes for transmission lines is an interesting topic for relay engineers and researchers [5]-[7].

Manuscript received $* * * * * *$; revised H*****; accepted $^{* * * * * * *}$; Date of publication $* * * * * *$; date of current version $* * * * * *$. This work was supported in part by the State Scholarship Fund of China Scholarship Council under Grant 202007000135, and in part by the Postgraduate Scientific Research \& Innovation Competition Project of Southwest Jiaotong University under Grant 2020KCJS17. Paper no. TPWRD-00030-2021. (Corresponding author: Xiaoyang Tong.)

G. Zhang and X. Tong are with the School of Electrical Engineering, Southwest Jiaotong University, Chengdu, Sichuan 611756, China (e-mail: gxzhang@my.swjtu.edu.cn; xytong@swjtu.cn).

Q. Hong and C. D. Booth are with the Department of Electronic and Electrical Engineering, University of Strathclyde, Glasgow G1 1XW, U.K. (e-mail: q.hong@strath.ac.uk; campbell.d.booth@strath.ac.uk).

Color versions of one or more of the figures in this letter are available online at http://ieeexplore.ieee.org.

Digital Object Identifier
Currently, many new schemes have been proposed for pilot protection in power systems. Protection schemes based on the waveform similarity principle have been one of the most used in $\mathrm{AC}$ transmission line [8]-[10], [16], [17], [22]-[24], DC transmission lines [11], [18]-[21], and transformers [12]-[15], because of their explicit and straightforward principle, and rapid and reliable fault detection. For instance, the authors of [10] utilized fault-induced traveling waves to design ultra-high-speed directional protection criteria based on the correlation between incremental quantities; with this, internal and external faults of an $\mathrm{AC}$ transmission line could be discriminated. The authors of [11] proposed a pilot protection scheme based on the similarity measure of traveling waves for VSC-HVDC transmission lines. However, since the traveling wave obtained is sensitive to transient signals and requires high sampling rates and synchronization requirements, there are some difficulties when it comes to implementing these schemes in practice. The internal and external faults of protected electrical equipment can be identified quickly by utilizing distance measure algorithms such as the discrete Fréchet distance [12], Euclidean distance [13], and Hausdorff distance (HD) [14], [15], [24]. A suitable threshold should be set to normalize the sampling data utilized in these methods. The Cosine Similarity (CS) [16]-[19] and Pearson Correlation Coefficient (PCC) [20]-[23] are widely used measure indices to calculate the similarity between two time-series signals. The effectiveness of these waveform similarity-based methods has been validated through extensive testing under different fault types, fault distances, fault resistances, noise, and CT saturation.

In [25], a secure phasor-based phase comparison (PC) protection scheme was proposed to identify internal or external faults based on the current phase angle difference between the two ends of a transmission line. However, it limits the speed of fault clearance because of the need for a data window to process phasor estimation using full-cycle Fourier transform. Time-domain PC protection schemes can operate faster than conventional phasor-based ones because they do not require the phasor estimation process. The methods developed for time-domain PC protection schemes can be conventionally classified into two groups: half-wave-based [26] and full-cycle-based [27]. The half-wave-based PC protection schemes only analyze the positive half cycles of the current signals while neglecting the negative half cycles, which may cause PC protection operation delays of approximately half a cycle depending on the fault inception angle. To eliminate this delay, the full-cycle-based PC protection scheme has been 
proposed to identify internal faults when the high level remains for more than one-quarter of a cycle. These schemes require a counter to estimate the pulse length, which is activated in case of consistent polarity of the currents measured at both terminals of the transmission line. However, a reverse outlier may cause the count to reset and thus lead to a refused protection relay operation.

The main drawback of the above schemes is that their reliability is significantly affected by outliers, which can be induced by measurement device failures, interfered measurement scans, and cyber-attacks. Since false data transmitted to a protection relay would result in a false tripping signal, some studies have focused on developing outlier detection or identification methods for the relay's cyber-security. An improved HD-based pilot protection with high speed has been proposed [24] to eliminate false data by introducing a data pre-processing approach. In [28], [29], cyber-resilient relays were developed that can detect false data against current differential protection. The authors of [30] presented a learning-based framework for intrusion detection against line current differential relays. These works have focused on outlier detection in protection relays. However, regardless of the performance of outlier detection methods, outliers cannot be eliminated entirely; thus, there is scope to develop a robust protection algorithm to deal with undetected false data.

In this paper, a robust pilot protection scheme based on the Kendall's Tau coefficient (KTC) is proposed for AC transmission lines. The internal and external faults in a transmission line are discriminated on the basis of the KTC value between the refactoring currents, which are constructed using the currents at both terminals of the transmission line to eliminate the effect of CT saturation. The effectiveness of the proposed criterion against different fault types, distances, and resistances is verified. Moreover, its effectiveness against CT saturation, synchronization error, and noise is proven. The KTC is less sensitive to outliers than existing similarity methods such as CS-based and PCC-based methods, ensuring protection safety in the presence of outliers.

The rest of this paper is organized as follows: Section II analyzes the characteristics of current waveforms during internal and external faults, and the construction of refactoring currents. Section III discusses the principle of KTC and its subsequent implementation for robust transmission line protection. Next, in Section IV, the simulated results obtained under various fault conditions are provided to prove the performance of the proposed protection scheme, and finally, the conclusions of this paper are drawn in Section V.

\section{ANALYSIS OF FAULT CHARACTERISTICS}

To better understand the waveform similarity-based protection principles, a two-machine system is considered as shown in Fig. 1. We assume that the positive directions of the currents at the two terminals of the line are toward the protected transmission line.

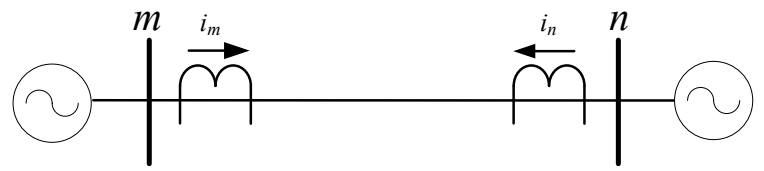

Fig.1. Single-phase diagram of a two-ended transmission line.

Ideally, the directions of the current waveforms $i_{m}$ and $i_{n}$ at the two terminals of the transmission line will be opposite and with the same magnitude, regardless of whether it is a normal condition or an external fault. When an internal fault occurs, currents $i_{m}$ and $i_{n}$ will flow toward the fault point. The directions of the current waveforms at the two terminals of the transmission line will be identical. However, a phase displacement between $i_{m}$ and $i_{n}$ may arise because of the charging currents; thereby, a reliable threshold should be used to ensure the protection security. Furthermore, the difference in the magnitudes of the current waveforms is typically significant depending on the fault point position. The following method can help eliminate the dissimilarity due to the magnitude difference between the current waveforms at the two terminals. At an instant $k$, the current waveforms can be expressed as:

$$
\begin{aligned}
& i_{r}(k)=i_{m}(k) \times i_{n}(k) \\
& i_{s}(k)=\left|i_{m}(k)\right| \times\left|i_{n}(k)\right|
\end{aligned}
$$

where $i_{r}(k)$ and $i_{s}(k)$ represent the refactoring currents having the same absolute value, i.e., $\left|i_{r}\right|=\left|i_{s}\right|$.

Let $X$ and $Y$ be two datasets with $N$ samples of the refactoring current $i_{r}$ and $i_{s}$, respectively. Given an instant $k$ they can be expressed as:

$$
\begin{aligned}
& X=\left[i_{r}(k-N+1), i_{r}(k-N), \ldots, i_{r}(k)\right] \\
& Y=\left[i_{s}(k-N+1), i_{s}(k-N), \ldots, i_{s}(k)\right]
\end{aligned}
$$

where $N$ is the number of samples per cycle.

The datasets obtained using the above approach can effectively deal with the problems related to the magnitude of the original current signals. Moreover, when an external fault occurs, the refactoring currents in the datasets $X$ and $Y$ exhibit opposite variation trends, whereas when an internal fault occurs, the refactoring currents in the datasets $X$ and $Y$ exhibit the same variation trends.

\section{Proposed Protection Scheme}

A pilot protection scheme using the $\mathrm{KTC}$ is proposed in this section. The similarity difference in the refactoring currents can be used as a protection criterion to identify the status of the transmission line under normal condition or in the event of external/internal faults.

\section{A. Principle of Kendall's Tau coefficient}

The similarity between two datasets is a significant notion in data mining. Various similarity measure methods, such as HD-based, CS-based, and PCC-based methods, have been used to distinguish between two datasets. However, these methods are highly sensitive to outliers, which can have a tremendous effect on the similarity measure. This means that including outliers in the measurements can lead to misleading results when using the above methods. To address this issue, we introduce the KTC [31], which is a robust similarity measure method, because it is based on simply the rank order as opposed 
to the metric value and thus limits outliers to a moderate level.

Let $\left(x_{1}, y_{1}\right),\left(x_{2}, y_{2}\right), \ldots,\left(x_{N}, y_{N}\right)$ be $N$ observations of the datasets $X$ and $Y$. The concordant definition for any pair observations $\left(x_{i}, y_{i}\right)$ and $\left(x_{j}, y_{j}\right)$ is given as follows:

For $\forall j>i$, where $i, j=1,2, \ldots, N$, if the following relationship holds,

$$
\left(x_{j}-x_{i}\right)\left(y_{j}-y_{i}\right)>0
$$

then the observations $\left(x_{i}, y_{i}\right)$ and $\left(x_{j}, y_{j}\right)$ are concordant, i.e., the sort order of the pair observations is in agreement.

If the following relationship is satisfied,

$$
\left(x_{j}-x_{i}\right)\left(y_{j}-y_{i}\right)<0
$$

Then the observations $\left(x_{i}, y_{i}\right)$ and $\left(x_{j}, y_{j}\right)$ are discordant, i.e., the sort order of the pair observations is not in agreement.

If the following relationship holds,

$$
\left(x_{j}-x_{i}\right)\left(y_{j}-y_{i}\right)=0
$$

then the observations $\left(x_{i}, y_{i}\right)$ and $\left(x_{j}, y_{j}\right)$ are independent. $\left\{\left(x_{i}, y_{i}\right)\right.$, $\left.\left(x_{j}, y_{j}\right)\right\}$ is consider to be tied if $x_{i}=x_{j}$ or $y_{i}=y_{j}$. When tied pairs arise in a dataset, the Tau-b statistic adjusts for these ties to keep the coefficient in the range $[-1,1]$. The formula for Kendall's tau-b is [32]:

$$
\tau=\frac{N_{c}-N_{d}}{\sqrt{N_{0}-T_{X}} \sqrt{N_{0}-T_{Y}}}
$$

where,

$$
\begin{gathered}
N_{0}=N(N-1) / 2 \\
T_{X}=\sum_{p=1}^{P} t_{p}\left(t_{p}-1\right) / 2 \\
T_{Y}=\sum_{q=1}^{Q} u_{q}\left(u_{q}-1\right) / 2
\end{gathered}
$$

where $N_{0}$ is the total number of unique unordered pairs that can be constructed for a sample size of $N$; $T_{X}$ is the total number of unique unordered pairs tied only on the dataset $X ; T_{Y}$ is the total number of unique unordered pairs tied only on the dataset $Y$. $t_{p}$ is the number of tied values in the $p^{\text {th }}$ group of ties for the dataset $X ; u_{q}$ is the number of tied values in the $q^{\text {th }}$ group of ties for the dataset $Y ; P$ and $Q$ represent the number of groups of ties for the datasets $X$ and $Y$, respectively. $N_{c}$ is the number of concordant pairs, and $N_{d}$ is the number of discordant pairs. An explicit expression for $N_{c}-N_{d}$ is written as,

$$
N_{c}-N_{d}=\sum_{1 \leq i<j \leq N} \operatorname{sign}\left(\left(x_{i}-x_{j}\right)\left(y_{i}-y_{j}\right)\right)
$$

where,

$$
\operatorname{sign}\left(\left(x_{i}-x_{j}\right)\left(y_{i}-y_{j}\right)\right)=\left\{\begin{aligned}
1, & \left(x_{j}-x_{i}\right)\left(y_{j}-y_{i}\right)>0 \\
0, & \left(x_{j}-x_{i}\right)\left(y_{j}-y_{i}\right)=0 \\
-1, & \left(x_{j}-x_{i}\right)\left(y_{j}-y_{i}\right)<0
\end{aligned}\right.
$$

Essentially, the KTC represents the ordinal association between two datasets. The calculated value of the coefficient $\tau$ is within the range of $[-1,1]$, where $\tau=1$ implies a perfect agreement between the two datasets $X$ and $Y$ and $\tau=-1$ indicates that one dataset is the reverse of the other. When the coefficient $\tau$ is approximately zero, the datasets $X$ and $Y$ are said to be independent. The properties of the KTC are utilized for the pilot protection in this work. The details of the scheme are provided in the following subsection.

\section{B. Protection Criterion based on Kendall's Tau Coefficient}

1) Protection Criterion: Based on the analysis of the fault characteristics mentioned in Section II, the refactoring currents in the datasets $X$ and $Y$ are found to be similar during the internal fault. Ideally, the KTC between two datasets identical in shape and trend is concordant and equal to "+1," which satisfies:

$$
\tau(X, Y)=1
$$

where $\tau(X, Y)$ represents the KTC between two datasets $X$ and $Y$ composed of the refactoring currents $i_{r}$ and $i_{s}$ obtained using Eqs. (1) and (2), respectively.

Under the normal condition and external fault, the refactoring currents in the datasets $X$ and $Y$ are dissimilar. Ideally, the KTC between two datasets similar in shape and opposite in trend is discordant and equal to " -1 ." Thus,

$$
\tau(X, Y)=-1
$$

From the above analysis, it can be concluded that during internal and external faults, the KTC between the datasets $X$ and $Y$ indicates the faulty or healthy status of the transmission line. Therefore, protection criteria can be constructed on the basis of the evident difference in the KTC values, which can help distinguish internal and external faults. If the following condition holds, an internal fault occurs:

$$
\tau(X, Y)>\tau_{\text {set }}
$$

where $\tau_{\text {set }}$ is a predetermined threshold value for the protection.

2) Threshold Setting: Interference factors, such as phase shift, capacitance current and communication error, affect the integrity of the current sampling values obtained from the two terminals of the transmission line and influence the judgment of similarity. Because the magnitude of the current waveforms can be eliminated by the proposed approach, mentioned in Section II, only the effect of the angle difference between the two datasets $X$ and $Y$ on the KTC needs to be considered. Therefore, the performance of the proposed algorithm would deteriorate when there are differences in the current angles between the two terminals.

Since the magnitude influence due to various unfavorable factors has been eliminated by the refactoring currents constructed in Section II, only the effect of the angle difference at the two terminals is the main factor that needs to be considered. A phase difference within $90^{\circ}(\tau=0)$ between the currents at the two terminals is a reliable boundary because once this tipping point is crossed, an internal fault can be misinterpreted as an external fault and vice versa. The threshold for the protection should be able to avoid fake operations due to a phase difference under various circumstances. We recommend setting the value to zero to balance the effect of the angle difference on both the internal and external faults under various unfavorable factors.

\section{Practical Implementation of the Proposed Scheme}

The main idea is to measure the similarity between two datasets exhibiting fault characteristics, which can be described using the KTC, to identify internal or external faults. Fig. 2 shows the flowchart of the proposed scheme, and the detailed step-by-step implementations are summarized as follows: 


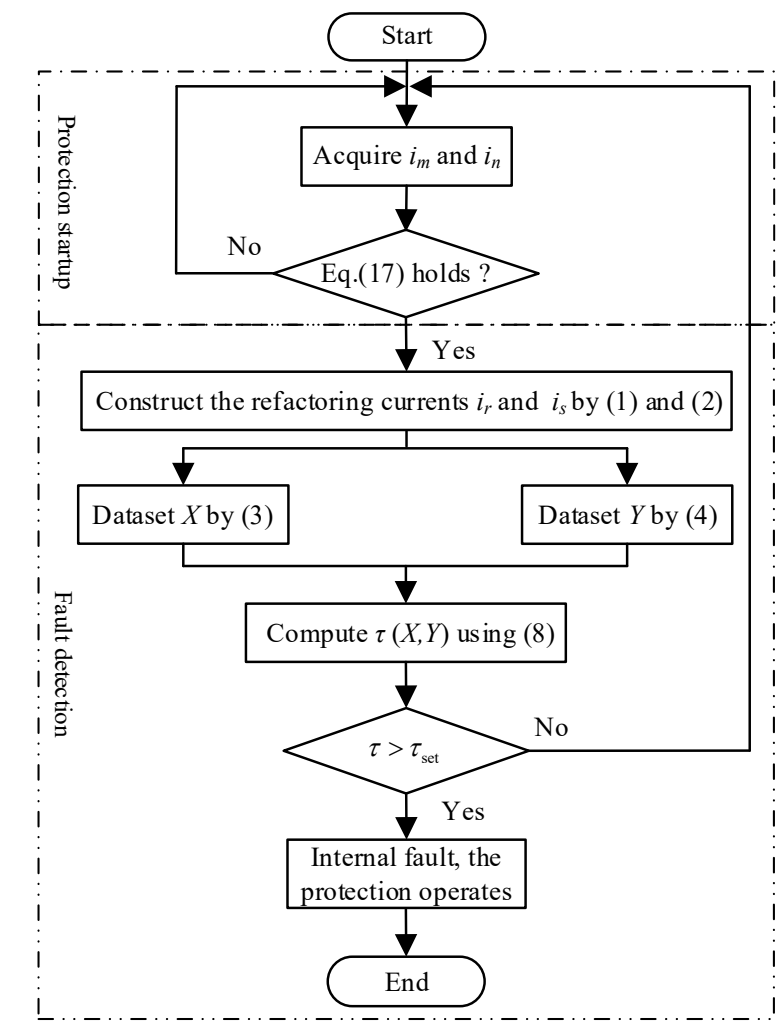

Fig.2. Flowchart of the protection criterion.

1) The measured currents at the two terminals of the transmission line are gathered. Subsequently, the following criterion in each phase $(\varphi)$ is checked for detecting the presence of a fault condition [33].

$$
|| i_{\varphi}(k)-i_{\varphi}(k-N)|-| i_{\varphi}(k-N)-i_{\varphi}(k-2 N)|| \geq 0.2 I_{n}
$$

where $I_{n}$ is the rated current. The threshold of $0.2 I_{n}$ is a predetermined value from experience [16], which has a good sensitivity to distinguish normal and faulty conditions under various conditions with an appropriate speed of action of about $1 \mathrm{~ms}$. Once the above condition is satisfied for any three successive samples starting from the instant $k$, the algorithm proceeds to the following step.

2) After detecting a faulty condition, the measured currents $i_{m}$ and $i_{n}$ are gathered, and Eqs. (1) and (2) are solved to construct the refactoring currents $i_{r}$ and $i_{s}$ to eliminate the dissimilarity due to the magnitude difference between them. Finally, two datasets $X=\left[i_{r}(k-N+1), i_{r}(k-N), \ldots\right.$, $\left.i_{r}(k)\right]$ and $Y=\left[i_{s}(k-N+1), i_{s}(k-N), \ldots, i_{s}(k)\right]$ with $N$ samples at an instant $k$ are obtained using Eqs. (3) and (4), respectively.

3) Calculations for the proposed scheme are performed to identify whether the fault is internal or external. For the instant $k$ at which the faulty condition is detected, the KTC between the two datasets $X$ and $Y$ is calculated using Eq. (8) considering a full-cycle sliding window with the first window being sample number $[k-N+1]$ to $[k]$.

4) The KTC value is assessed, where the transmission line, whose protection criterion Eq. (16) is satisfied, would be determined as the faulty line and would issue a tripped signal for isolating the fault. Otherwise, the fault is external, and the protection is reset.

\section{Simulation Results}

An IEEE 10 -generator 39 -bus system operating at $345 \mathrm{kV}$, as shown in Fig. 3, is utilized to evaluate the performance of the proposed pilot protection based on the KTC under various fault conditions. The studied test system is developed using the PSCAD/EMTDC and the parameters can be referred from Ref. [34]. The current signals are sampled at a rate of $3 \mathrm{kHz}$ at a base frequency of $60 \mathrm{~Hz}$. The sampling data in the full-cycle window are used for computing the protection criterion and are moved forward once a new sampling value is taken.

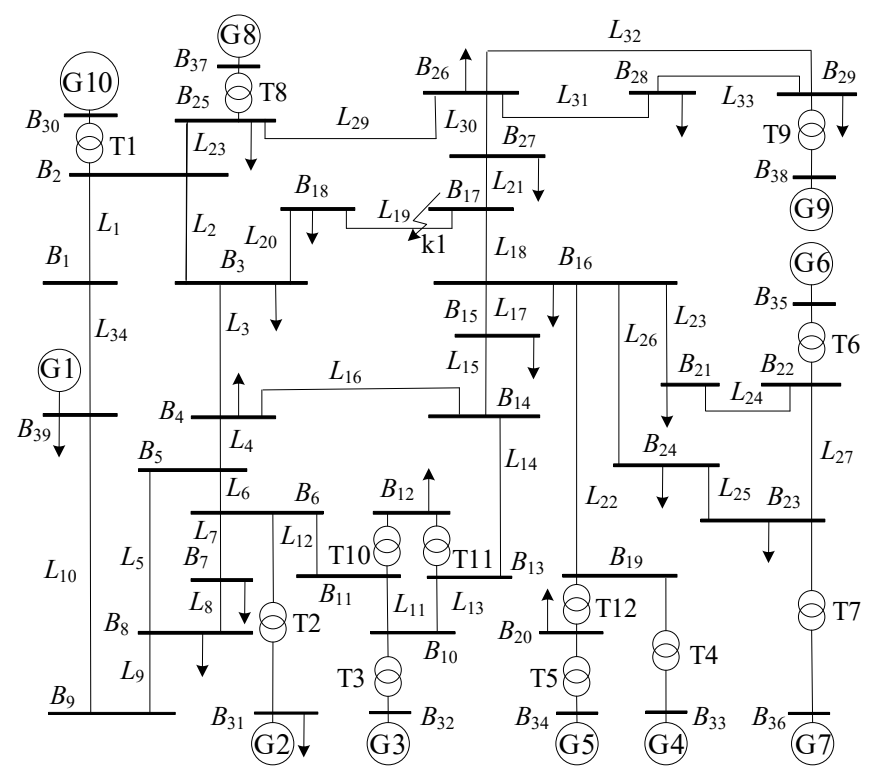

Fig.3. IEEE 10-generator 39-bus system.

\section{A. Results for External and Internal Faults}

1) Effects of Fault types: A double-line-to-ground fault with fault resistance of $0.01 \Omega$ is simulated on line $L_{19}$ at $95 \%$ away from Bus B18 at $0.3 \mathrm{~s}$, denoted by k1 as shown in Fig. 3. Figs. 4(a) and (b) show the original currents in the healthy line $L_{18}$ and faulty line $L_{19}$, respectively. Next, the refactoring currents $i_{r}$ and $i_{s}$ in the healthy line $L_{18}$ and faulty line $L_{19}$ are obtained as shown in Figs. 4(c) and (d), respectively.

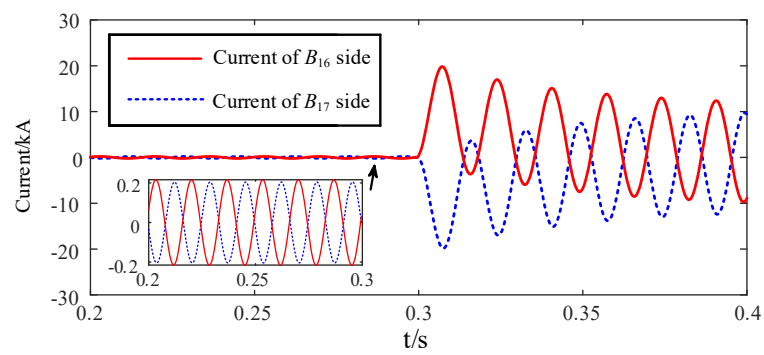

(a)

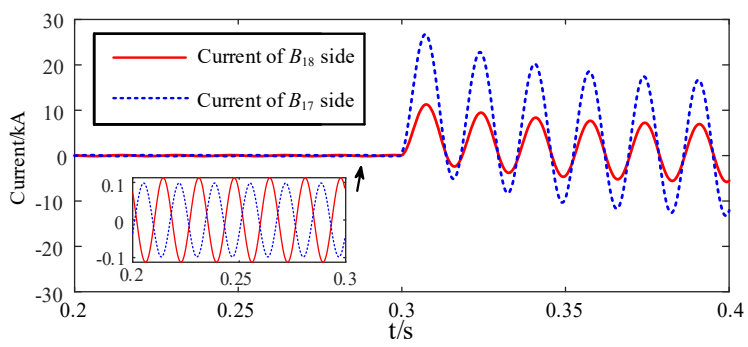

(b) 


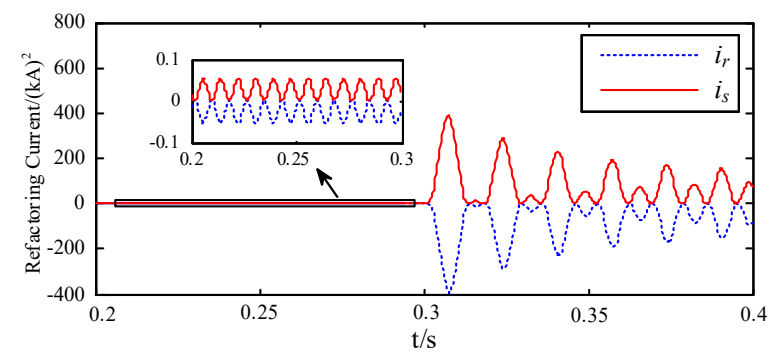

(c)

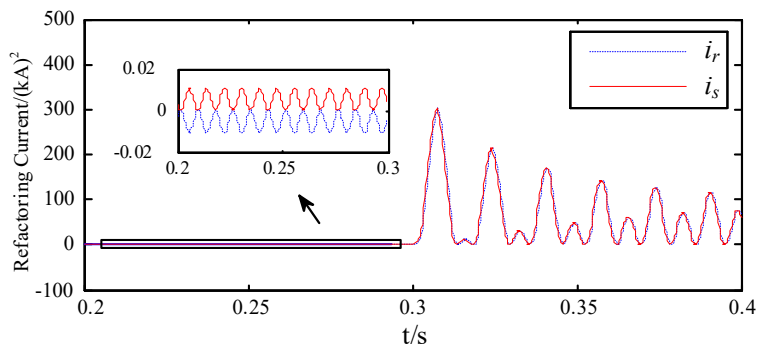

(d)

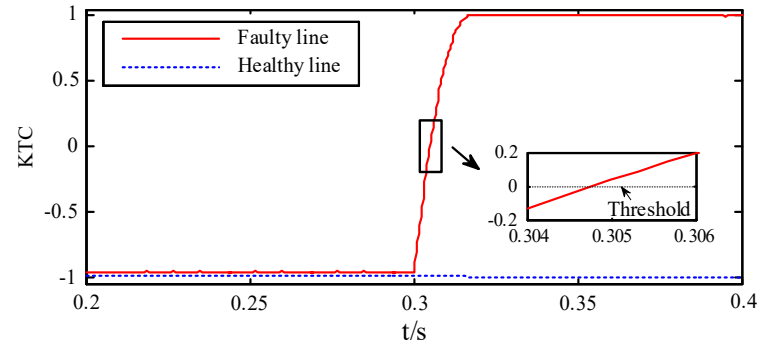

(e)

Fig.4. Original currents in (a) healthy line $L_{18}$, (b) faulty line $L_{19}$; and the refactoring currents in (c) healthy line $L_{18}$, (d) faulty line $L_{19}$; and (e) operating quantities of KTC-based protection.

Fig. 4(e) shows the KTC values of the faulty line $L_{19}$ exceeds the threshold 0 within $5 \mathrm{~ms}$ after a fault occurs and gradually stabilizes around 1 . The KTC values of the adjacent healthy line $L_{18}$ are far below the threshold. The similarity is strengthened in the healthy line when the sets $X$ and $Y$ contain the steady-state current waveforms entirely after $0.3 \mathrm{~s}$, and the value is maintained around -1 .

Figs. 5(a)-(c) show the results of the single-line-to-ground, line-to-line, and three-phase fault at the fault point $\mathrm{k} 1$, respectively. Fig. 5 shows that the proposed method has a significant margin of operation for correctly identifying the faulty line within $6 \mathrm{~ms}$ regardless of the fault type.

2) Effects of Fault Distance: The fault distance is an essential factor in evaluating the protection performance. To this end, the single-line-to-ground faults are simulated on line $L_{19}$ with a fault resistance of $100 \Omega$, and the fault distance is varied from $10 \%$ to $95 \%$. Fig. 6 shows the results for the healthy line $L_{18}$ and faulty line $L_{19}$ at different fault distances. In this figure, the values of the KTC at different fault distances are almost equal to -1 for the healthy line $L_{18}$ and stabilize close to 1 for the faulty line $L_{19}$. Therefore, we can conclude that the performance of the proposed protection scheme is unaffected by the fault distance.

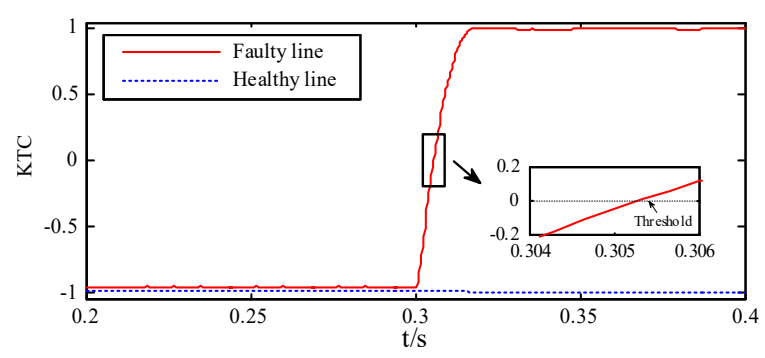

(a)

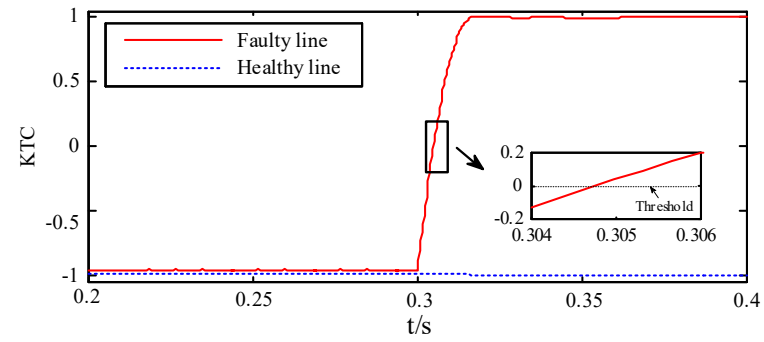

(b)

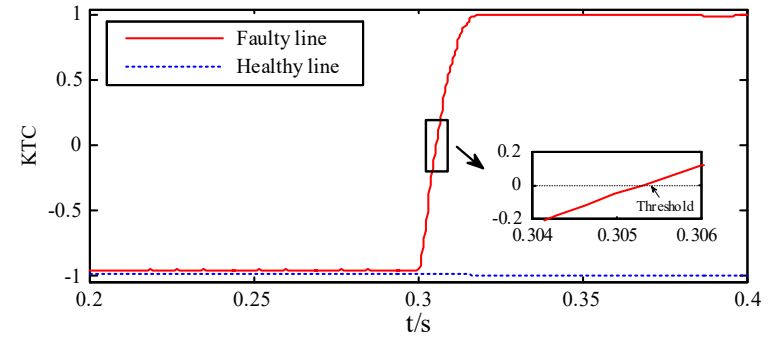

(c)

Fig.5. Operating quantities of KTC-based protection for healthy line $L_{18}$ and faulty line $L_{19}$ : (a) single-line-to-ground fault, (b) line-to-line fault, (c) three-phase fault.

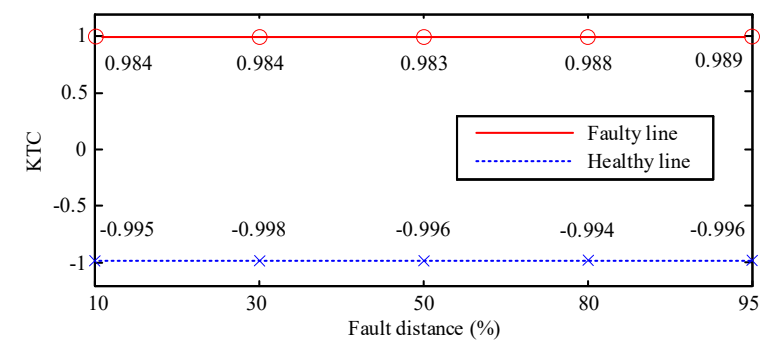

Fig.6. Operating quantities of KTC-based protection for healthy line $L_{18}$ and faulty line $L_{19}$ at different fault distances.

3) Effects of Fault Resistance and Load: To analyze the effect of fault resistance, the single-phase grounding faults with different fault resistances are set on the line $L_{19} 10 \%$ away from Bus $B_{18}$ at 0.3 s. Fig. 7 shows the calculation results of line $L_{19}$ under different fault resistances.

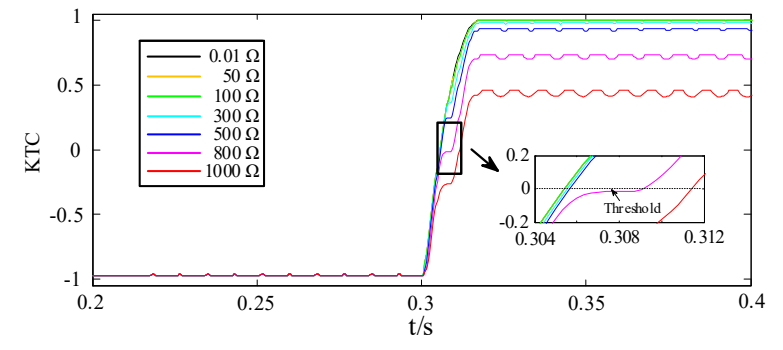

Fig.7. Operating quantities of KTC-based protection under different fault resistances. 
The proposed scheme can identify faults within $6 \mathrm{~ms}$ when the fault resistance is no more than $500 \Omega$, as shown in Fig. 9 . With the increase in the fault resistance from $0.01 \Omega$ to $1000 \Omega$, the calculated value of the KTC reduces. However, the operation of the proposed protection criterion is unaffected; a fault with a resistance of $1000 \Omega$ can be identified with the lowest action speed of $12 \mathrm{~ms}$.

To verify the influence of loads with different power factors, a single-line-to-ground fault is created at the middle of the transmission line $L_{19}$ with a fault resistance of $100 \Omega$. The KTC values for the healthy line $L_{18}$ and faulty line $L_{19}$ are computed after varying the power factors of the loads at node $B_{18}$ and tabulated in Table I. From Table I, we find that the performance of the proposed algorithm is unaffected by the power factor loads.

TABLE I

EFFECT OF POWER FACTOR LOADS ON THE PROPOSED METHOD

\begin{tabular}{ccccc}
\hline \hline \multirow{2}{*}{ Power factor } & \multicolumn{2}{c}{ Load } & & \\
\cline { 2 - 3 } & $\begin{array}{c}\text { Active } \\
\text { (MW) }\end{array}$ & $\begin{array}{c}\text { Reactive } \\
\text { (MVAR) }\end{array}$ & $L_{18}$ & $L_{19}$ \\
\hline 0.98 & 158 & 30 & -0.994 & 0.983 \\
0.8 & 129 & 32 & -0.996 & 0.995 \\
0.6 & 96 & 64 & -0.998 & 0.995 \\
0.4 & 64 & 96 & -0.995 & 0.986 \\
0.2 & 32 & 129 & -0.993 & 0.978 \\
\hline \hline
\end{tabular}

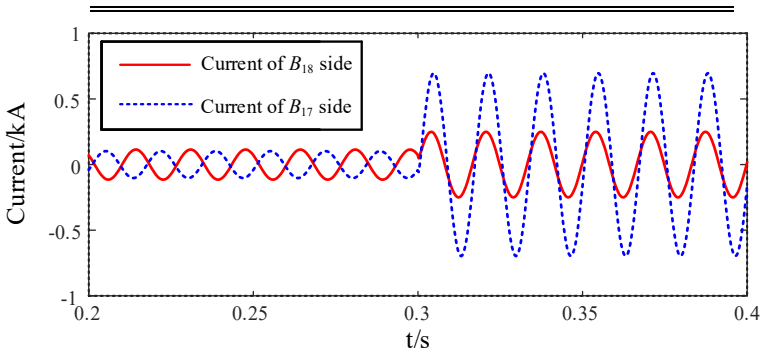

(a)

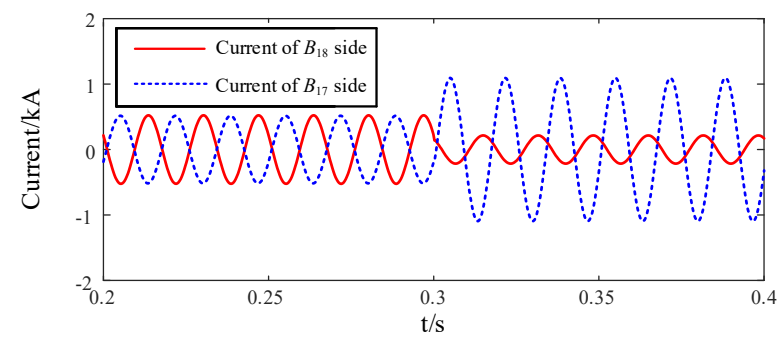

(b)

Fig.8. Waveforms of currents at the two terminals of the transmission line $L_{19}$ under (a) original and (b) thrice the load flow value in node $B_{18}$.

The proposed method is unaffected by the high fault resistance because the fault current effect predominates over the low loading levels. Normally, for a $300-400 \mathrm{kV}$ network, the maximum resistance investigated is around $100 \Omega$. The performance during max assumed loading is acceptable. Fig. 8 shows the current waveforms for the original and thrice the load flow value in node $B_{18}$ during a single-line-to-ground fault with a resistance of $300 \Omega$ at the middle of the transmission line $L_{19}$. With the increase in the load flow, the load current contribution is more significant than the fault current. Under this circumstance, the currents in the $B_{18}$ side that should have been considered as entering the protection zone, as shown in Fig. $8(\mathrm{a})$, are interpreted as leaving the protection zone, as shown in Fig. 8(b), which characterizes external faults or normal condition. Therefore, the system loads influence the performance of the proposed algorithm when the load current predominates the fault current during fault condition; Nevertheless, this can be overcome by employing incremental currents to eliminate the effects of the system loads.

\section{B. Effect of CT Saturation}

To verify the effect of CT saturation on the performance of the proposed method, a single-line-to-ground fault with a fault resistance of $100 \Omega$ at the fault point k1 in the line $L_{19} 95 \%$ away from bus $B_{18}$ is set at 0.3 s. Fig. 9 shows the current waveforms with CT saturation in the case of both internal and external faults.

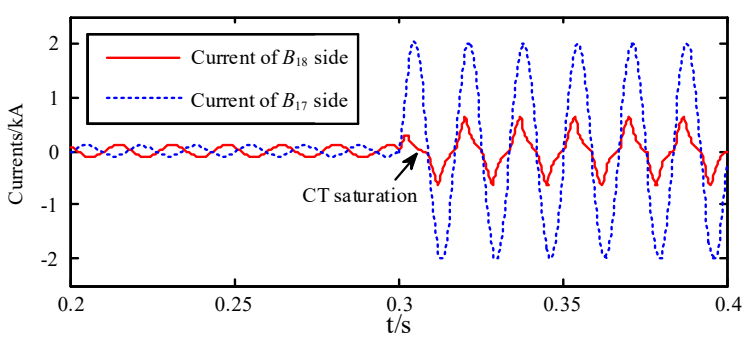

(a)

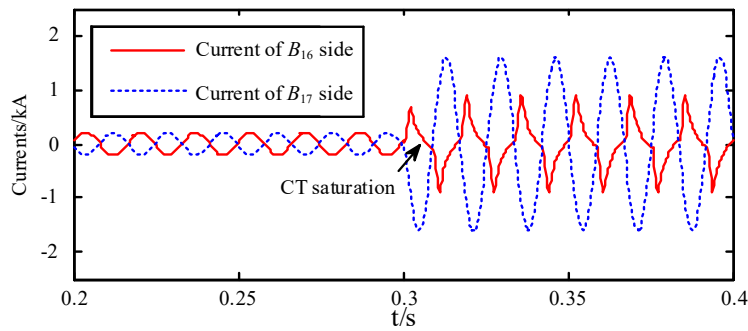

(b)

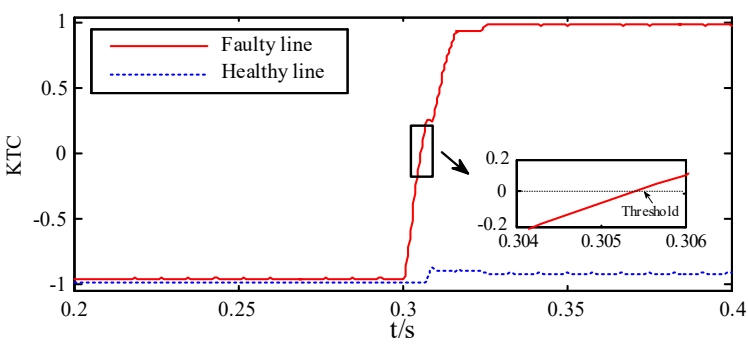

(c)

Fig. 9. Current waveforms with CT saturation under (a) internal fault in line $L_{19}$; (b) external fault in line $L_{18}$; and (c) operating quantities of KTC-based protection for both lines.

Figs. 9(a) and (b) show that the magnitude of the current waveform on the CT saturation side is lower than the true one and has a clipping edge phenomenon, leading to the maloperation of the conventional differential protection. Although the waveforms of the original currents $i_{m}$ and $i_{n}$ are distorted due to $\mathrm{CT}$ saturation, the waveform characteristics of the refactoring currents constructed using Eqs. (1) and (2) are largely unaffected by the CT saturation because the magnitude difference between the two waveforms is eliminated. Therefore, as shown in Fig. 9(c), both the internal and external faults with CT saturation can be correctly identified using the proposed protection criterion, with an action speed of $<6 \mathrm{~ms}$.

\section{Effects of Synchronization Error}

Current measurements are sometimes not synchronized 
because of a synchronization error or when a slow communication channel is utilized for pilot protection. To verify the influence of synchronization delay on the performance of the proposed method, a single-line-to-ground fault with a $100 \Omega$ fault resistance is simulated at the fault point k1. Figs. 10(a) and (b) show the calculated results of the proposed protection algorithm for the faulty and healthy lines considering different communication delays, respectively.

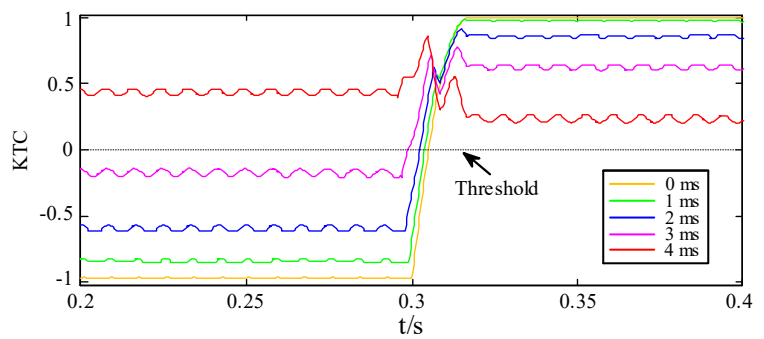

(a)

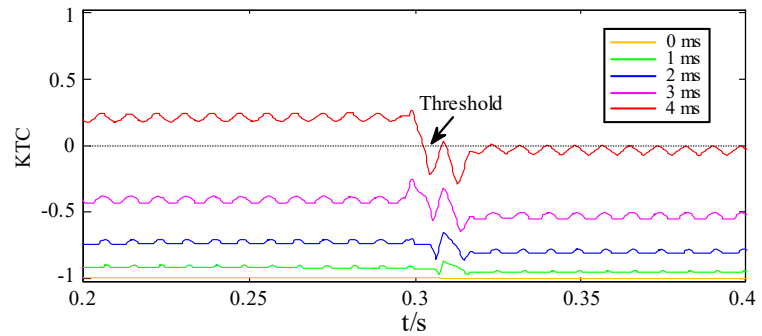

(b)

Fig.10. Operating quantities of KTC-based protection under different communication delays: (a) faulty line $L_{19}$; (b) healthy line $L_{18}$.

The results show that the proposed algorithm does not require strict communication synchronization and can reliably identify internal faults in the event of a communication delay within $3 \mathrm{~ms}$. Notably, as mentioned in Section III.B, a misjudgment between the faulty and healthy lines occurs when the phase angle difference between the current waveforms at the terminals is $90^{\circ}$ (4.167 ms for a $60 \mathrm{~Hz}$ system). However, the maximum delay would be less than the theoretical one considering the already existing angle difference between the current waveforms due to the capacitance current and phase shift.

\section{Sensitivity to Noise}

The measurement accuracy is susceptible to noise, and it could be included in the current measurements. To validate the proposed method considering noise interference, Gaussian noise with magnitudes of $10,20,30,40$, and $50 \mathrm{~dB}$ are superimposed onto the current signals at the two terminals of the transmission line. Figs. 11(a) and (b) show the calculated KTC values for the faulty and healthy lines with different signal-to-noise ratios (SNRs) under a single-line-to-ground fault with a $100 \Omega$ fault resistance occurring at the fault point $\mathrm{k} 1$ after $0.3 \mathrm{~ms}$, respectively.

Although the noise changes only the magnitude of the measured current, the polarity and direction of the current with small magnitude may be changed. Therefore, as shown in Fig. 11, a certain amount of Gaussian noise effects normal conditions more than fault conditions. Furthermore, for both the faulty and healthy lines, the KTC values are insensitive to the noise when the SNR is $>20 \mathrm{~dB}$, and the protection criterion can ensure a reliably operation, thus verifying the excellent anti-noise performance of the proposed method.

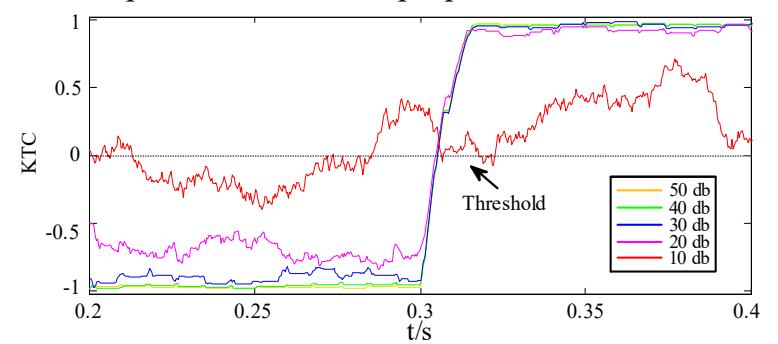

(a)

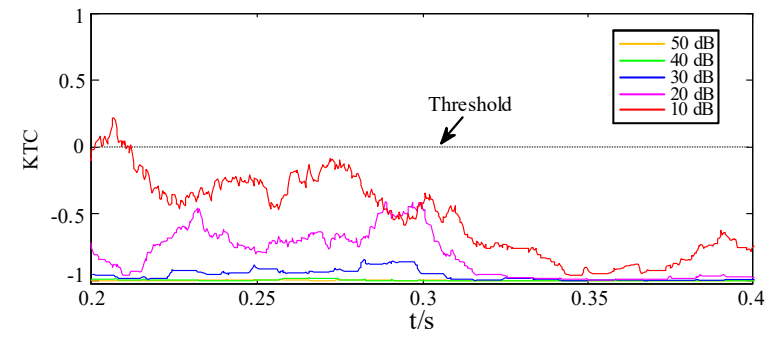

(b)

Fig. 11. Operating quantities of KTC based protection under different SNR values: (a) faulty line $L_{19}$; (b) healthy line $L_{18}$.

\section{E. Influence of Outliers and Comparison work}

Conventionally, outliers can be due to measurement device failures and interfered measurement scans. Furthermore, bad measurements can be introduced by an attacker to manipulate digital relays maliciously. Regardless of the effectiveness of software and hardware measures, the outliers cannot be eliminated completely; thus, it is necessary to test the impact of outliers on the protection algorithm. A single-line-to-ground fault with a fault resistance of $300 \Omega$ is induced at the midpoint of line $L_{19}$. Figs. 12 (a) and (b) show the current waveforms of the faulty line $L_{19}$ and healthy line $L_{18}$ with outliers, respectively.

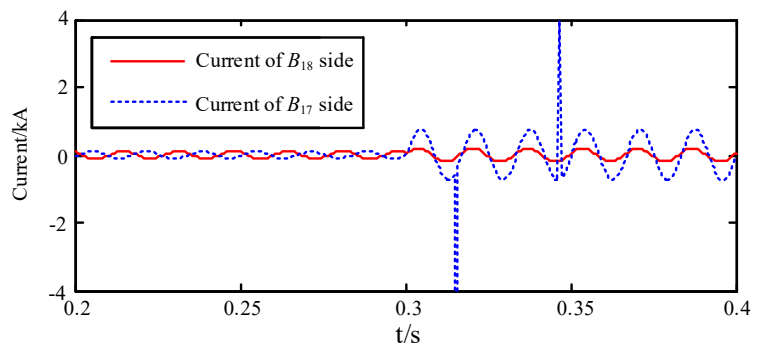

(a)

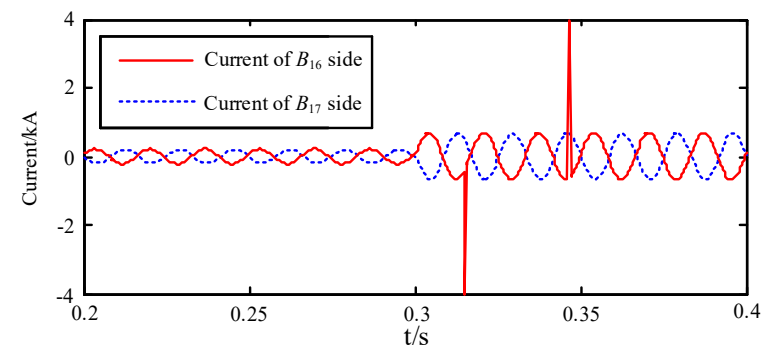

(b)

Fig.12. Current waveforms with outliers (a) faulty line $L_{19}$; (b) healthy line $L_{18}$

For comparison, CS-based [16]-[19] and PCC-based methods [20]-[23] are chosen. As shown in Fig.13, the outliers having the same direction as the current waveform do not affect 
the calculated results. Figs. 13(a) and (b) present the operating quantities of the CS-based and PCC-based methods, respectively. Both the methods are sensitive to the second reverse outliers, and the operating quantities fluctuate significantly, whether it is a faulty line or a healthy line, resulting in the malfunction of the protection criterion. Fig. 13(c) shows the operating quantities of the proposed algorithm. The reverse outliers slightly reduce the calculated value of the faulty line, whereas they slightly increase for the healthy line, but both deviate away from the threshold with a sufficient margin, ensuring protection safety.

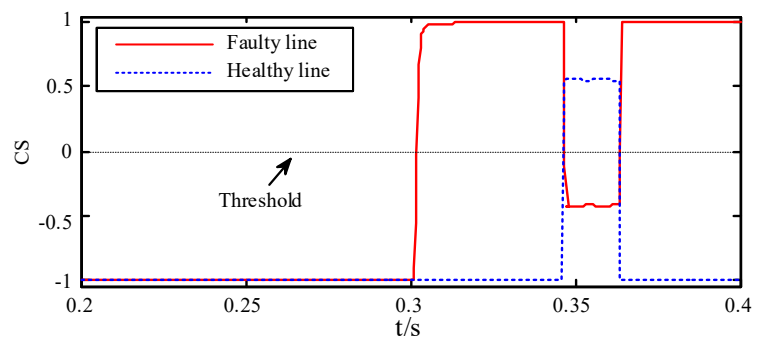

(a)

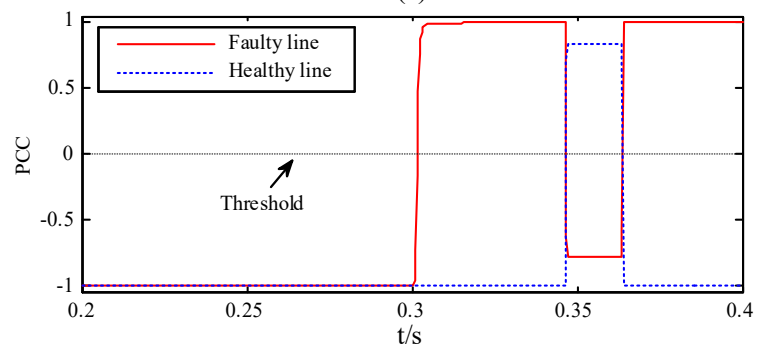

(b)

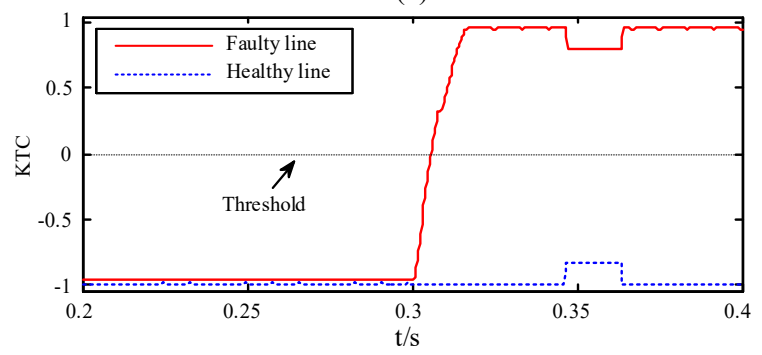

(c)

Fig.13. Operating quantities of waveform similarity-based protection for both lines: (a) CS-based method; (b) PCC-based method; (c) Proposed method.

The influence of the value size of outliers on the three protection methods is given in Table II. As the magnitude of the abnormal value increases, the calculated values obtained using the CS-based and PCC-based methods deviate from the expected value and become more significant, thus leading to maloperation. In comparison, the proposed method is insensitive to outliers, and the KTC remains constant when the magnitude of the abnormal value increases to a certain extent. The proposed method can correctly identify the fault since the outliers are limited to a moderate level by utilizing the rank order instead of the metric value.

TABLE II

INFLUENCE OF THE VALUE SIZE OF OUTLIERS ON THE PROTECTION METHODS

\begin{tabular}{lcccccc}
\hline \hline \multirow{2}{*}{ Method } & \multirow{2}{*}{ Normal } & \multicolumn{5}{c}{ Outliers } \\
\cline { 3 - 7 } & & 0.5 & 1 & 2 & 3 & 4 \\
\hline CS-based & 0.9999 & 0.9070 & 0.6738 & 0.1241 & -0.2736 & -0.5142 \\
PCC-based & 0.9997 & 0.7937 & 0.3540 & -0.3347 & -0.6438 & -0.7846 \\
Proposed & 0.9771 & 0.8792 & 0.8188 & 0.8188 & 0.8188 & 0.8188 \\
\hline \hline
\end{tabular}

\section{F. Two-machine System Operating at $500 \mathrm{kV}$}

The higher the voltage level of the transmission line, the longer the line length and the larger the capacitive current compared to the one with a lower voltage level. To evaluate the effects of the line length and capacitive current on the proposed algorithm, a $50 \mathrm{~Hz}$ two-machine system operating at $500 \mathrm{kV}$ is tested in PSCAD/EMTDC and the parameters can be referred from Ref. [35].

1) Effect of Line Length: The line length is changed to short, medium, and long transmission lines to evaluate its effect on the proposed method. Fig. 14 shows that before the introduction of the fault, although the long transmission line produces higher KTC values than the shorter one due to the existence of higher distributive capacitance, the values are far less than the threshold. Moreover, the KTC values after introducing the fault tend to be stable and are much greater than the threshold. Therefore, we can conclude that the line length has little effect on the protection performance.

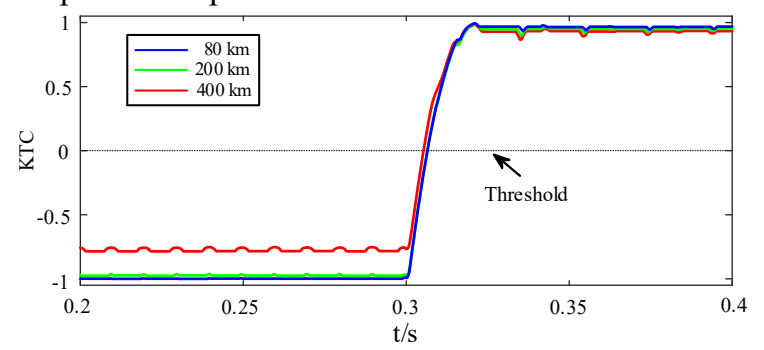

Fig.14. Operating quantities of KTC with different line lengths.

2) Effect and Analysis of Capacitive Current: To analyze the influence of capacitive current on the proposed method, a single-line-to-ground fault is created at the center of a $200 \mathrm{~km}$ line for the two-machine system at $0.3 \mathrm{~s}$ with distributive capacitance parameters set to twice, thrice, four times, five times and six times the original value. Fig. 15 shows the KTC values in the presence of the above line.

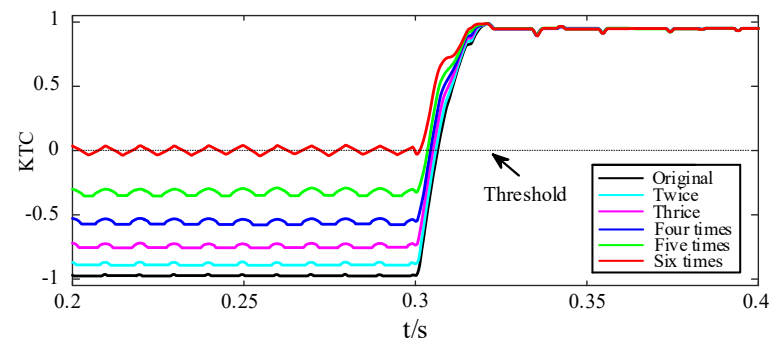

Fig.15. Operating quantities of KTC with different line-distributed capacitance.

With increase in the line distributive capacitance parameters, the KTC value increases under the normal condition (before 0.3 $\mathrm{s}$ ), as shown in Fig. 15. However, the KTC value is unaffected and is greater than 0.9 during fault conditions. The capacitive current of the transmission line has a more negative effect on the protection under normal conditions than fault conditions. The load current is more susceptible to the capacitive current because the fault current is usually much higher than the load current. Specifically, the capacitive current of the transmission line has a negative effect on the protection under normal conditions because of the phase difference due to the influence of the capacitive current. However, the discharge current of the line-distributive capacitor after the introduction of the fault 
does not affect the waveform change direction of the fault current or the polarity, as the fault current has a more dominant effect than the capacitive current.

Capacitive currents may cause magnitude and phase shift of the currents on both sides. Since the magnitude effect of the original signals is eliminated in this work, the calculated results are unaffected by the magnitude differences. The phase shift due to the capacitive current depends on the $\mathrm{X} / \mathrm{R}$ ratio of the line and system equivalents [26]. For higher $X / R$ values, the capacitive current affects mostly the magnitudes of the terminal currents. For lower X/R values (highly resistive impedances), the capacitive current affects the phase relationship. Therefore, the effect of capacitor current for a high-voltage system with large $\mathrm{X} / \mathrm{R}$ values can be bypassed by a threshold since they have a higher safety boundary.

A problem occurs when a weak system feeds an external fault through the protected line. The charging current may be higher than the actual inductive current generated by the weak source, and the former would invert the current measured at the weak terminal, which means that the phase difference between the currents at the two terminals is $180^{\circ}$, misleading the protection to detect an internal fault in the healthy lines [26]. This drawback can be mitigated by compensating for the line capacitive currents from the measured ones, ensuring line protection security under weak infeed.

\section{CONCLUSION}

This study developed a KTC-based robust protection method for AC transmission lines. The KTC was computed using the proposed refactoring currents, which could effectively deal with the problems related to the magnitude of the original current signals. Extensive simulations demonstrated that the proposed protection algorithm has good performance under different fault types, fault distances, and fault resistances and that it is robust against CT Saturation, synchronization error, and noise. Moreover, the proposed criterion was found to be insensitive to outliers compared with the existing criteria, ensuring protection safety in case outliers cannot be eliminated by the detection method. In the future work, the proposed KTC-based pilot protection would be combined with incremental currents and capacitive currents compensation approaches to overcome the effects of system loads and line capacitances.

\section{REFERENCES}

[1] J. Suonan, K. Liu, and G. Song, "A Novel UHV/EHV Transmission-Line Pilot Protection Based on Fault Component Integrated Impedance," IEEE Trans. Power Del., vol. 26, no. 1, pp. 127-134, Jan. 2011.

[2] S. AsghariGovar and H. Seyedi, "A Novel Transfer Matrix-Based Approach for Pilot Protection of Hybrid Transmission Lines Considering HIF Location," IEEE Trans. Power Del., vol. 35, no. 4, pp. 1749-1757, Aug. 2020.

[3] T. G. Bolandi, H. Seyedi, S. M. Hashemi, and P. S. Nezhad, "Impedance-Differential Protection: A New Approach to Transmission-Line Pilot Protection," IEEE Trans. Power Del., vol. 30, no. 6, pp. 2510-2518, Dec. 2015.

[4] G. Chen, Y. Liu, and Q. Yang, "Impedance Differential Protection for Active Distribution Network," IEEE Trans. Power Del., vol. 35, no. 1, pp. 25-36, Feb. 2020.
[5] D. T. Dantas, E. L. Pellini, and G. Manassero, "Time-Domain Differential Protection Method Applied to Transmission Lines," IEEE Trans. Power Del., vol. 33, no. 6, pp. 2634-2642, Dec. 2018.

[6] S. Liu, L. L. Zhang, C. Fu, and L. Jiang, "A New Two-Port Network Model-Based Pilot Protection for AC Transmission Lines," IEEE Trans. Power Del., vol. 35, no. 2, pp. 473-482, Apr. 2020.

[7] L. Chen et al., "Remedial Pilot Main Protection Scheme for Transmission Line Independent of Data Synchronism," IEEE Trans. Smart Grid, vol. 10, no. 1, pp. 681-690, Jan. 2019.

[8] L. Zheng, K. Jia, T. Bi, Z. Yang and Y. Fang, "A Novel Structural Similarity Based Pilot Protection for Renewable Power Transmission Line," IEEE Trans. Power Del., vol. 35, no. 6, pp. 2672-2681, Dec. 2020.

[9] K. Jia, Z. Yang, L. Zheng, Z. Zhu, and T. Bi, "Correlation-Based Protection for Transmission Line Connected to Wind Farms," IEEE Trans. Ind. Informat., doi: 10.1109/TII.2020.3018499

[10] A. Lei, X. Dong, and V. Terzija, "An ultra-high-speed directional relay based on correlation of incremental quantities," IEEE Trans. Power Del., vol. 33, no. 6, pp. 2726-2735, Dec. 2018.

[11] Y. Wang, Z. Hao, B. Zhang, and F. Kong, "A pilot protection scheme for transmission lines in VSC-HVDC grid based on similarity measure of traveling waves," IEEE Access, vol. 7, pp. 7147-7158, 2019.

[12] H. Weng, S. Wang, Y. Wan, X. Lin, Z. Li, and J. Huang, "Discrete Fréchet distance algorithm based criterion of transformer differential protection with the immunity to saturation of current transformer," Int. J. Electr. Power Energy Syst., vol. 115, pp. 105449, Feb. 2020.

[13] T. Zheng; X. Liu, J. Wei; M. A. Khan, and X. Wang, "Protection scheme for turn-to-turn faults of magnetically controlled shunt reactor based on waveform similarity comparison," Electr. Power Syst. Res., vol. 177, pp. 105980, Dec. 2019.

[14] H. Weng, S. Wang, X. Lin, Z. Li, and J. Huang, "A novel criterion applicable to transformer differential protection based on waveform sinusoidal similarity identification," Int. J. Electr. Power Energy Syst., vol. 105, pp. 305-314, Feb. 2019.

[15] S. Ding, X. Lin, Z. Zhang, Z. Li, L. Chen, and H. Weng, "A novel Hausdorff distance based restrain criterion for zero-sequence differential protection of converter transformer," Int. J. Electr. Power Energy Syst., vol. 105, pp. 753-764, Feb. 2019.

[16] A. N. R. L. Sirisha and A. K. Pradhan, "Cosine Similarity Based Directional Comparison Scheme for Subcycle Transmission Line Protection," IEEE Trans. Power Del., vol. 35, no. 5, pp. 2159-2167, Oct. 2020.

[17] L. Zheng, K. Jia, T. Bi, Y. Fang, and Z. Yang, "Cosine Similarity Based Line Protection for Large Scale Wind Farms," IEEE Trans. Ind. Electron., doi: 10.1109/TIE.2020.2998756

[18] M. Farshad, "A Pilot Protection Scheme for Transmission Lines of Half-Bridge MMC-HVDC Grids Using Cosine Distance Criterion," IEEE Trans. Power Del., doi: 10.1109/TPWRD.2020.3001878

[19] K. Jia, C. Wang, T. Bi, R. Zhu, and Z. Xuan, "Transient current waveform similarity based protection for flexible DC distribution system," IEEE Trans. Ind. Elect., vol. 66, no. 12, pp. 9301-9311, Dec. 2019

[20] Y. Qin, M. Wen, Y. Bai, Y. Wang, X. Wang, and Z. Fang, "A novel pilot protection scheme for HVDC lines based on waveform matching," Int. J. Electr. Power Energy Syst., vol. 120, pp. 106028, Sep.2020.

[21] Y. Zhang, Y. Li, J. Song, X. Chen, Y. Lu, and W. Wang, "Pearson correlation coefficient of current derivatives based pilot protection scheme for long-distance LCC-HVDC transmission lines," Int. J. Electr. Power Energy Syst., vol. 116, 105526, Sep. 2020.

[22] X. Wang, M. Wen, J. Zheng, Y. Chen, Y. Qin, and Y. Ma, "A novel directional relay for AC lines close to the HVDC installation," Int. J. Electr. Power Energy Syst., vol. 118, pp. 105726, Jun. 2020.

[23] K. Jia, Y. Li, Y. Fang, L. Zheng, T. Bi, and Q. Yang, "Transient current similarity based protection for wind farm transmission lines," Appl. Energy, vol. 225, pp. 42-51, Sep. 2018.

[24] L. Chen et al., "Similarity comparison based high-speed pilot protection for transmission line," IEEE Trans. Power Del., vol. 33, no. 2, pp. 938948, Apr. 2018

[25] T. P. Hinge and S. S. Dambhare, "Secure Phase Comparison Schemes for Transmission-Line Protection Using Synchrophasors," IEEE Trans. Power Del., vol. 30, no. 4, pp. 2045-2054, Aug. 2015. 
[26] B. Kasztenny, I. Voloh, and E. A. Udren, "Rebirth of the phase comparison line protection principle," 59th Annu. Conf. Protect. Relay Eng., TX, USA, 2006.

[27] E. J. S. L. Junior, F. V. Lopes, and K. M. Silva, "Time-domain application of superimposed quantities in the phase comparison transmission line protection scheme," J. Eng. Vol. 15, pp. 1360-1364, Sep., 2018.

[28] A. Ameli, A. Hooshyar, and E. F. El-Saadany, "Development of a Cyber-Resilient Line Current Differential Relay," IEEE Trans. Ind. Informat., vol. 15, no. 1, pp. 305-318, Jan. 2019.

[29] A. Ameli, A. Hooshyar, E. F. El-Saadany, and A. M. Youssef, "An Intrusion Detection Method for Line Current Differential Relays," IEEE Trans. Inf. Forensics Security, vol. 15, pp. 329-344, 2020.

[30] A. Ameli, A. Ayad, E. El-Saadany, M. Salama, and A. Youssef, "A Learning-Based Framework for Detecting Cyber-Attacks Against Line Current Differential Relays," IEEE Trans. Power Del., doi: 10.1109/TPWRD.2020.3017433

[31] M. G. Kendall, "A new measure of rank correlation," Biometrika, vol. 30, no.1/2, pp. 81-93, Jun. 1938.

[32] M. G. Kendall and J. D. Gibbons, Rank Correlation Methods, 5th ed. New York: Oxford Univ. Press, 1990.

[33] A. Jalilian, M. T. Hagh, and S. M. Hashemi, "An innovative directional relaying scheme based on postfault current," IEEE Trans. Power Del., vol. 29, no. 6, pp. 2640-2647, Dec. 2014.

[34] I. Hiskens, "IEEE PES Task Force on Benchmark Systems for Stability Controls," Tech. Rep., Nov. 2013.

[35] X. Tong, H. Wen, "A novel transmission line fault detection algorithm based on pilot impedance," Electr. Power Syst. Res., vol. 179, pp. 106062 , Feb. 2020.

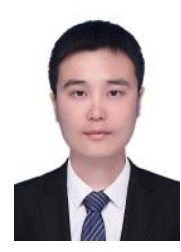

Guangxiao Zhang (Student Member, IEEE) is currently working toward the $\mathrm{Ph} . \mathrm{D}$. degree in electrical engineering with the School of Electrical Engineering, Southwest Jiaotong University, Chengdu, Sichuan, China. He is also a Sponsored Visiting Researcher in the Department of Electronic \& Electrical Engineering, University of Strathclyde, Glasgow, U.K.. His research interests include the application of wide-area measurements, state estimation and artificial intelligence in power systems, power system protection, fault location, and fault isolation.

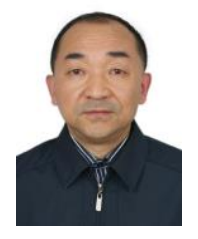

Xiaoyang Tong received the B.Sc., M.Sc., and Ph.D. degrees from the Southwest Jiaotong University, Chengdu, Sichuan, China, in 1993, 1996, and 2007, respectively. He is currently an Associate Professor with the School of Electrical Engineering, Southwest Jiaotong University. His research interests include wide-area protection, power system fault diagnosis, energy internet, and smart substation.

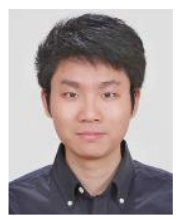

Qiteng Hong (S'11-M'15) received the B.Eng. (Hons.) and Ph.D. degrees in electronic and electrical engineering from the University of Strathclyde, Glasgow, U.K., in 2011 and 2015, respectively. $\mathrm{He}$ is currently a Lecturer (Strathclyde's Chancellor's Fellow) with the University of Strathclyde. His main research interest includes power system protection and control of future networks with high penetration of renewable generation. Dr. Hong is a Regular Member CIGRE Working Group B5.50, IEEE Working Group P2004. He was the Technical Lead at the CIGRE UK Next Generation Network (2014-2019) and the Secretary of IET Scotland Southwest Committee (2015-2018).

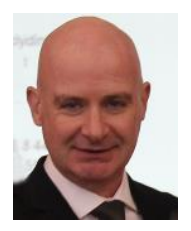

Campbell D. Booth received the B.Eng. and Ph.D. degrees in electrical and electronic engineering from the University of Strathclyde, Glasgow, U.K., in 1991 and 1996, respectively. He is currently a Professor and Head of the Department for Electronic and Electrical Engineering, University of Strathclyde. His research interests include power system protection, plant condition monitoring and intelligent asset management, applications of intelligent system techniques to power system monitoring, protection, and control, knowledge management, and decision support systems. 